\title{
Upward Drawing on the Plane Grid Using Less Ink (Extended Abstract)
}

\author{
Guy-Vincent Jourdan ${ }^{1}$, Ivan Rival ${ }^{2}$, and Nejib Zaguia ${ }^{3}$ \\ 1 IRISA, Campus Universitaire Beaulieu \\ 35042 Rennes Cédex France \\ jourdanQirisa,fr \\ 2 Department of Computer Science, University of Ottawa \\ Ottawa K1N 6N5 Canada \\ rivalecsi.uottawa.ca \\ ${ }^{3}$ Department of Computer Science, University of Ottawa \\ Ottawa KIN 6N5 Canada \\ zaguialcsi.uottawa.ca
}

\begin{abstract}
Any upward drawing $\mathcal{D}(P)$ on a two-dimensional integer grid $\mathcal{I}$, of an ordered set $P$, has completion $\bar{P}$ with an upward drawing $\mathcal{D}(\bar{P})$ on a two-dimensional integer grid $\overline{\mathcal{I}}$ such that the total edge length of $\mathcal{D}(\bar{P})$ does not exceed the total edge length of $\mathcal{D}(P)$. Moreover, by (possibly) translating vertices, there is an upward drawing $\mathcal{D}(P)$ on $\mathcal{I}$ such that $\overline{\mathcal{I}}=\mathcal{I}$.

Thus, any integer grid embedding of a two-dimensional ordered set can be extended to a planar upward drawing of its completion, on the same integer grid, without increasing the total edge length.
\end{abstract}

\section{Introduction}

Can we combine aesthetic standards of readability with the economy achieved by using less ink? [Tufte (1983)] formulates the "data-ink ratio" according to which a large share of the ink on a graphic should present data-information. [Aeschlimann and Schmid (1992)] seem to be the first to consider this problem explicitly, giving heuristcs, algorithms, and examples. For upward drawings of ordered sets there is a basis for optimism highlighted by the fact that, although the covering graph of a subset $Q$ of an ordered set $P$, may be a subgraph of the covering of $P$, and, therefore, have shorter total edge length, it need not be a subgraph of the covering graph of $P$. Indeed, the total edge length of $Q$ may be much more than the total edge length of an ordered set of which it is a subset (with the induced order). Cf. Figure 1.

Terminologically, we speak of three items :

- upward drawing $\mathcal{D}(P)$;

- order completion $\bar{P}$;

- edge length $e(\mathcal{D}(P))$. 
It is customary and convenient to render an ordered set by an upward drawing according to which the elements of the ordered set $P$ are drawn on a surface, traditionally a plane, as disjoint small circles, arranged in such a way that, for $a, b \in P$, the circle corresponding to $a$ is higher than the circle corresponding to $b$ whenever $a>b$ and an arc, monotonic with respect to a fixed direction, usually south to north, and straight, is drawn to join them just if $a$ covers $b$ (that is, for each $x \in P, a>x \geq b$ implies $x=b$ ). We say that $a$ is an upper cover of $b$ or $b$ is a lower cover of $a$, and write $a \succ b$ or $b \prec a$. These arcs are drawn, of course, to avoid the incidence of any other circle on it (to avoid unwanted comparabilities) and, moreover, when possible, to avoid intersections, too (except where two arcs meet at a circle). For our purposes, a straight line segment is drawn joining the circles corresponding to $a$ and $b$, if $a \succ b$ in $P$. Thus, the upward drawing, itself, avoids "nonessential" edges, that is, edges corresponding to comparabilities derivable from the transitivity condition. To this extent, the upward drawing is already calculated to minimize the number of edges in a graphical rendering.

Loosely speaking, the completion $\bar{P}$ of an ordered set $P$ is the smallest lattice containing $P$. The completion is a representation that has found application in several areas : in geographical information systems (cf. [Franzosa, Perry, Saalfeld, Wohlgemuth (1994)] the completion $\bar{P}$ is often easier to read and to draw, hence making easier the identification of geographical units corresponding to the elements of $P$; in the study of planarity of lattices and of dimension three orders [Kelly (1977)]. More precisely, for each $S \subseteq P$, set $S^{+}=\{x \in P: x \geq s$ for every $s \in S\}$ and $S_{-}=\{x \in P: x \leq s$ for every $s \in S\}$. Then the map $\nu$ which associates to each subset $S$ of $P, \nu(S)=\left(S^{+}\right)_{-}$is a closure operator on $P$ and the mapping $a \longrightarrow \nu(a)$ is an embedding of $P$ into the complete lattice $\{\nu(S): S \subseteq P\}$, ordered by inclusion, known variously as the normal completion, or Dedekind-MacNeille completion [MacNeille (1937)]. The completion of $P$ is $\bar{P}=\left\{S_{-}^{+}: S \neq \emptyset, S^{+} \neq \emptyset\right\}$, that is, $\bar{P}$ is this normal completion except for its top and/or bottom, unless $P$ already has top and/or bottom.

From our viewpoint these are the salient points about the completion:

(a) If $P$ has an upward drawing on a two-dimensional integer grid then so does its completion $\bar{P}$. An upward drawing of $P$ on a two-dimensional integer grid is an assignment of positive integers $x_{1}, x_{2}$ to its vertices $x \in P$ such that $\left(x_{1}, x_{2}\right)=x \leq y=\left(y_{1}, y_{2}\right)$ just if $x_{1} \leq y_{1}$ and $x_{2} \leq y_{2}$. Any such ordered set is two-dimensional in the usual sense that its order is the intersection of two linear extensions.

(b) The normal completion $\bar{P}$ is the smallest lattice containing $P$. The normal completion of an $n$-element ordered set may be exponential in size. Still, if it is two-dimensional, then the completion has size at most $O\left(n^{2}\right)$. At the same time there are several known output sensitive polynomial time algorithms to compute the completion [Ganter, Reuter (1991)], even on-line (cf. [Jourdan, Rampon, Jard (1994)]).

The length of an edge $a \succ b$ in an upward drawing $\mathcal{D}(P)$ rendered in $\mathbf{R}^{2}$ is its Euclidean length, computed between the centres of the circles corresponding to the elements $a, b$, and the edge length $e(\mathcal{D}(P))$ is the sum of the edge lengths of all of its edges in $\mathcal{D}(P)$. 


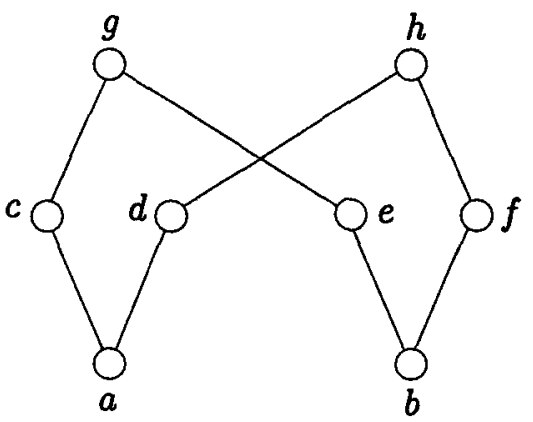

$Q_{1}$

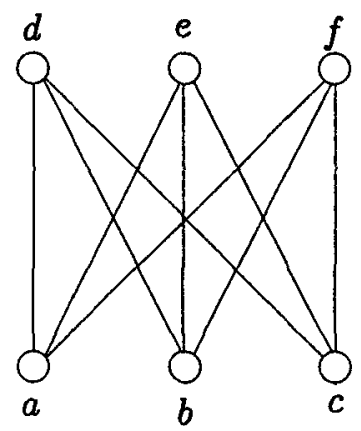

$$
Q_{2}=K_{3,3}
$$



$P_{1}$

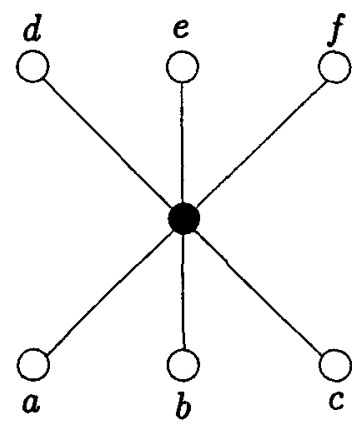

$$
P_{2}=\overline{K_{3,3}}
$$

Figure 1: The subset $Q_{1}$ has total edge length approximately 10 (normalized with edge length 1 for the edge $a \succ b$ ), which is less than the total edge length (approximately 16) of its completion $P_{1}$ of which it is a subset (and subgraph). On the other hand, the bipartite ordered set $Q_{2}=K_{3,3}$ has total edge length more than the edge length of its completion $P_{2}=\overline{K_{3,3}}$. 


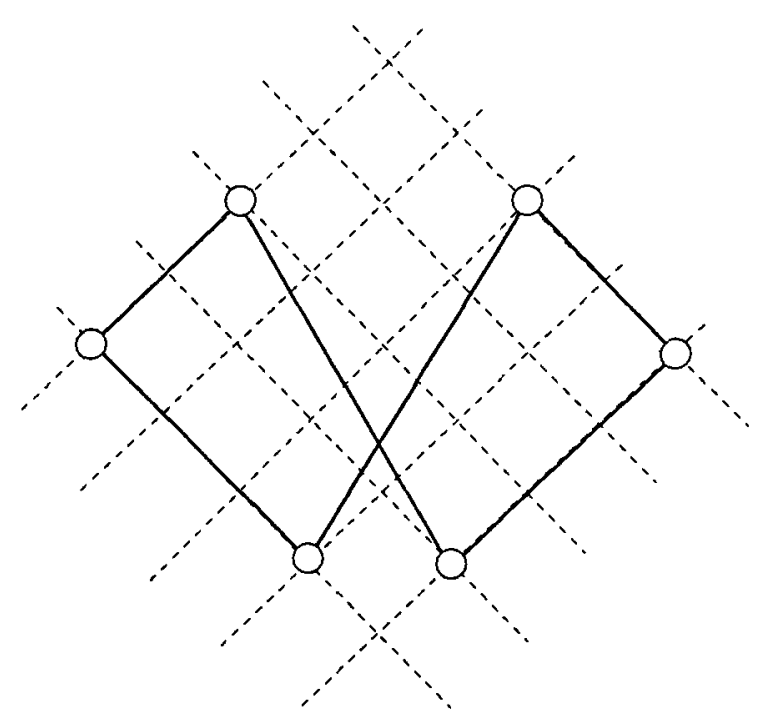

Figure 2: An integer grid upward drawing of an ordered set which cannot be extended to an upward drawing of its completion on the same integer grid, without exceeding the original edge length unless the positions of its vertices are changed, or the integer grid is changed.

Theorem 1 Any upward drawing $\mathcal{D}(P)$ on a two-dimensional integer grid $\mathcal{I}$, of an ordered set $P$, has completion $\bar{P}$ with an upward drawing $\mathcal{D}(\bar{P})$ on a two-dimensional integer grid $\overline{\mathcal{I}}$ such that

$$
e(\mathcal{D}(\bar{P})) \leq e(\mathcal{D}(P))
$$

Moreover, by (possibly) translating vertices of the upward drawing $\mathcal{D}(P)$ on $\mathcal{I}$, the upward drawing $\mathcal{D}(\bar{P})$ of $\bar{P}$ may be chosen on the same integer grid $\mathcal{I}$, that is, $\overline{\mathcal{I}}=\mathcal{I}$.

A few cautions about the proof.

- Although we can effect the construction sequentially, the elements of the completion cannot be added randomly - or even in the obvious way (see Figure 3).

- The two-dimensional grid customarily consists of two families of parallel lines, orthogonal to each other (cf. Figure 4). The centers of vertices of $P$ and of $\bar{P}$ are all located at intersections of these grid lines. The distances nevertheless are Euclidean distances along the line segment joining two vertices, not distances measured along the grid lines. In fact, we do not, at this writing, know whether our result holds true for any pair of families of parallel lines - not necessarily orthogonal. 

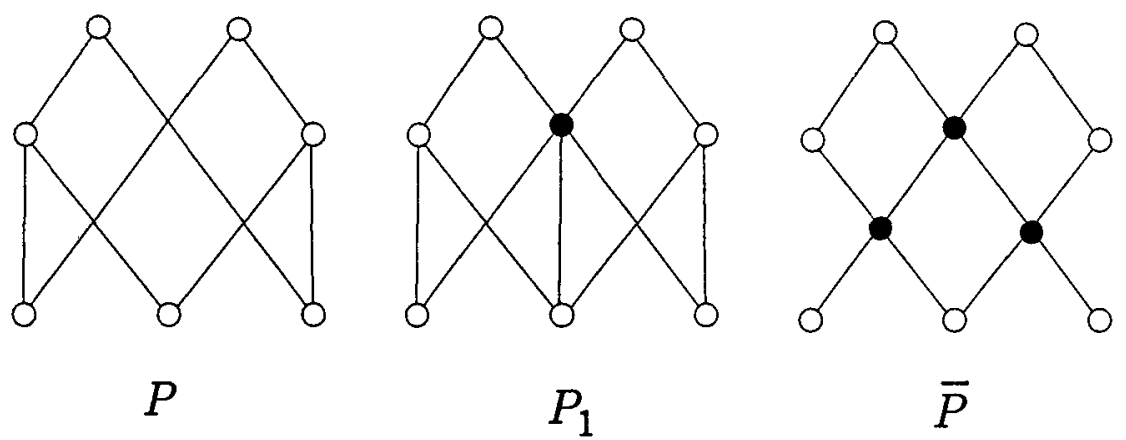

$\bar{P}$

Figure 3: The two-dimensional ordered set $P$ has completion $\vec{P}$ with shorter edge length, although the "intermediate" (one-element extension) $P_{1}$ has larger edge length.

- In fact, our proof shows that, except for a rather precise family of cases (in which equality occurs), $e(\mathcal{D}(\bar{P}))<e(\mathcal{D}(P))$.

- Although a two-dimensional ordered set $P$ may have an upward drawing, with its vertices located on a two-dimensional grid, the usual order of the grid need not induce the order of $P$, that is, its upward drawing is not an embedding into the grid (cf. Figure 2). In this case, its completion may have larger total edge length - if the vertices of $P$ remain unchanged in its completion $\bar{P}$.

- It is not always possible to extend an upward drawing on the two-dimensional integer grid to an upward drawing of its completion - without either changing the size of the integer grid, or changing the initial upward drawing (see Figure 2).

Is there any other way to save "ink"?

Corollary 1 Let $\mathcal{D}(P)$ be an upward drawing of $P$ on a two-dimensional integer grid, and let $\mathcal{D}(Q)$ be an upward drawing on the same integer grid, of an ordered set $Q$ containing $P$ with the integer grid coordinates of $P$ unchanged. Then

$$
e(\mathcal{D}(\bar{P})) \leq e(\mathcal{D}(Q))
$$

What is a "good" upward drawing? Of course, planarity is usually a desirable feature. For two-dimensional orders we can guarantee planarity without increasing total edge length. The obvious approach to add a vertex for every intersection of a pair of covering edges (see Figure 6) which, although it does not increase the total edge length, will have two distinct disadvantages :

- the number of vertices may be considerably larger than the size of the completion; 




$K_{3,3}$

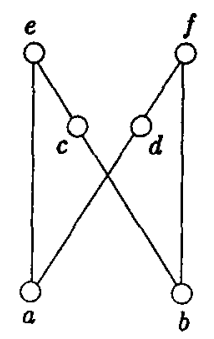

$P$

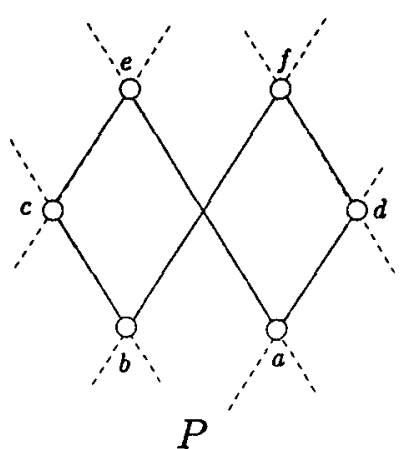

$P$



Figure 4: $K_{3,3}$ has an upward drawing on the plane grid. Its completion $\overline{K_{3,3}}$ in which it is order embedded does too. On the other hand, the ordered set $P$ (whose order can be induced by a twodimensional grid embedding) has an upward drawing whose order cannot be induced by such a grid embedding and whose total edge length is shorter than that of its completion. 
- the integer grid required may be considerably larger.

Corollary 2 Every two-dimensional ordered set can be embedded in its planar completion which itself has an upward drawing on an orthogonal grid - all without increasing its total edge length.

On the other hand, the number of vertices of this planar ordered set may increase. For instance, the completion of the $4 n$-element ordered set $P=\{(i, j)$ : $i=0$ and $j=0,1,2, \ldots, n$, or $j=0$ and $i=0,1,2, \ldots, n\} \cup\{(i, j): i=$ $n$ and $j=0,1,2, \ldots, n$, or $j=n$ and $i=0,1,2, \ldots, n\}$ is the $(n+1)^{2}$ grid $\{0,1,2, \ldots, n\} \times\{0,1,2, \ldots, n\}$ (cf. Figure 4). In this case, the total edge length of $P$ equals the total edge length of $\bar{P}$.

\section{Open Problems}

There is an evident parallel between our work and the "Steiner problem", which, for a given set of points seeks the "tree" with minimum possible total edge length, a problem with several known variants, all NP-complete (cf. [Garey, Johnson (1979)]). The Steiner ratio for a metric space is the largest lower bound for the ratio of lengths between a minimum Steiner tree and a minimum spanning tree on the same set of points in the metric space (cf. [Gao, Du, Graham], [Rao, Sadayappan, Hwang, Shor (1990)]). The Steiner ratio for a metric space is the largest lower bound for the ratio of lengths between a minimum Steiner tree and a minimum spanning tree on the same set of points in the metric space. The value of this ratio depends on the metric chosen.

Fix an ordered set $P$. Fix a upward drawing $\mathcal{D}(\mathcal{P})$ of $P$. We define the completion ratio of $P$ with respect to $\mathcal{D}(\mathcal{P})$ by

$$
\varepsilon(\mathcal{D}(\mathcal{P}))=\min _{\mathcal{D}(\overline{\mathcal{P}})} \frac{e(\mathcal{D}(\overline{\mathcal{P}}))}{e(\mathcal{D}(\mathcal{P}))}
$$

where $\mathcal{D}(\bar{P})$ is an upward drawing of the completion of $P$ in which the vertices corresponding to $P$ coincide with their position in $\mathcal{D}(P)$, and the completion ratio of $P$ by

$$
\varepsilon(P)=\min _{\mathcal{D}(\mathcal{P})} \varepsilon(\mathcal{D}(\mathcal{P}))
$$

If $P$ is a lattice then $\varepsilon(P)=1$. Evidently, $\varepsilon\left(K_{3,3}\right)<1$ (cf. Figure 1 or Figure 3 ) while, according to Figure $1, \varepsilon\left(Q_{1}\right)>1$.

Corollary 3 For any ordered set $P$ of dimension two

$$
\varepsilon(P) \leq 1
$$

Question $1^{1}$ Is there a constant $k$ such that, for any ordered set $P$,

$$
\varepsilon(P) \leq k \quad ?
$$

\footnotetext{
${ }^{1}$ Examples show that $k \geq 3$.
} 


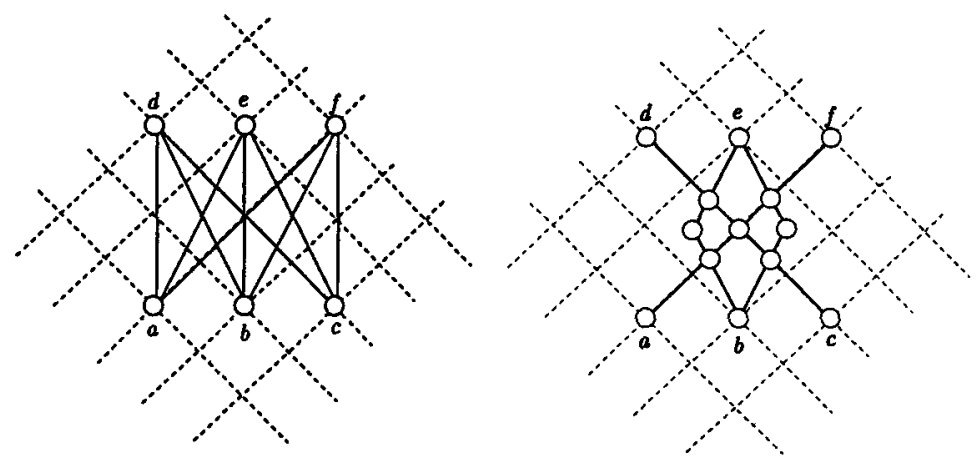

Figure 5: The upward drawing of $K_{3,3}$ on the integer grid can be extended to the upward drawing of a planar ordered set containing it, by adding vertices for intersection points of its covering edges. However, both the grid size and the number of vertices required may increase - by more than what is necessary for the completion $\overline{K_{3,3}}$.

Consider the hypercube $2^{n}$, that is, the ordered set of all subsets of an $n$-element set $\{1,2, \ldots\}$. Set $P \subset 2^{n}$ the subset of the singleton subsets $\{i\}$ and the one-element deleted subsets $\{1,2, \ldots, i-1, i+1, \ldots, n\}$. The completion of this $2 n$-element ordered set is $2^{n} \backslash\{\emptyset,\{1,2, \ldots\}\}$ and, since it has exponential size (in terms of the size of $P$ ) we may well expect that its edge length is a great deal larger than $e(\mathcal{D}(P))$ for any upward drawing $\mathcal{D}(P)$ of $P$.

Theorem 2 There is an upward drawing $\mathcal{D}\left(P_{n}\right)$ of the $2 n$-element ordered set $P_{n}=$ $\{\{i\},\{1,2, \ldots, i-1, i+1, \ldots, n\}: i=1,2, \ldots n\}$, ordered by set inclusion, and there is an upward drawing of its completion $\mathcal{D}\left(\overline{P_{n}}\right)$ containing $\mathcal{D}\left(P_{n}\right)$ such that $e\left(\mathcal{D}\left(\overline{P_{n}}\right)\right) \leq$ $\sqrt{2} e\left(\mathcal{D}\left(P_{n}\right)\right)$, that is, $\varepsilon\left(P_{n}\right)=\sqrt{2}$.

According to the construction of this theorem, there are edges with small length and others with quite large length.

Question 2 If every edge has length at least $k_{1}$ and at most $k_{2}\left(k_{1}, k_{2}\right.$ constants), is $\varepsilon\left(P_{n}\right)$ bounded?

Is there a "small" cover-preserving subset of the completion of $P$ (cf. [Lee, Liu, Nowakowski, Rival (1988)] ?

Question 3 Is there a constant $k$ such that, for any n-element ordered set $P$, there is an ordered set $Q \subseteq \bar{P}$ such that

(i) $P \subseteq Q$,

(ii) if $x \succ y$ in $Q$ then $x \succ y$ in $\bar{P}$,

(iii) $|Q|=O\left(n^{k}\right)$ ? 

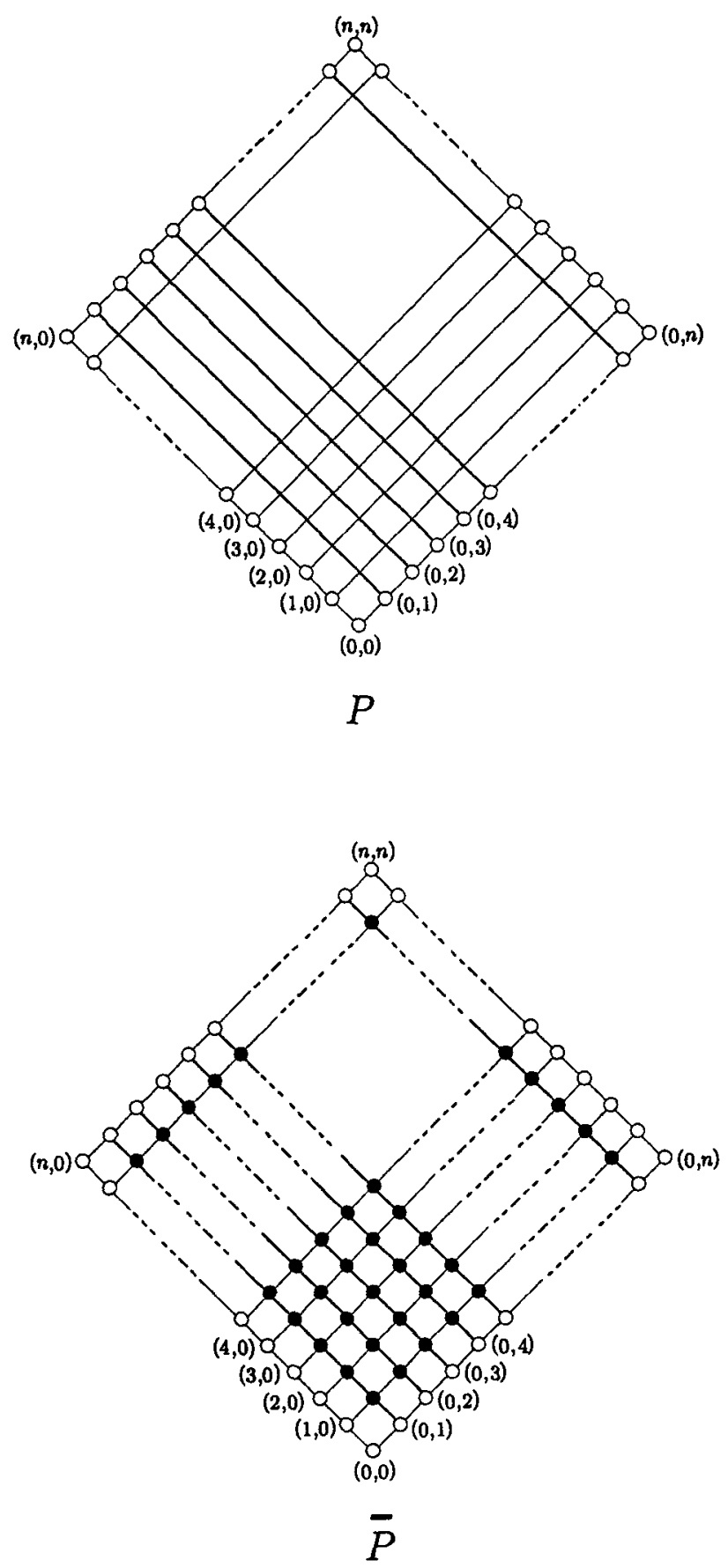

Figure 6: The two-dimensional grid $\mathrm{n} \times \mathrm{n}$ is the completion of the $4 n$-element ordered set $P$. The total edge length of $P$ equals the total edge length of $\bar{P}$. 


\section{References}

1. A. Aeschlimann and J. Schmid (1992) Drawing orders using less ink, ORDER 9, 5-13.

2. R. Franzosa, L. Perry, A. Saalfeld, and A. Wohlgemuth (1994) An outputsensitive polynomial-time algorithm for constructing the normal completion of a partially ordered set, preprint.

3. B. Ganter and K. Reuter (1991) Finding all closed sets : a general approach, ORDER 8, 283-290.

4. B. Gao, D.-Z. Du, and R. L. Graham, The tight lower bound for the Steiner Ratio in Minkowski planes.

5. M. R. Garey and D. S. Johnson (1979) Computers and Intractability : A guide to the Theory of NP-Completeness, Freeman, $\mathrm{x}+338$.

6. G.-V. Jourdan, J.-X. Rampon, and C. Jard (1994) Computing on-line the lattice of maximal antichains of posets, ORDER (to appear).

7. J. G. Lee, W.-P. Liu, R. Nowakowski, and I. Rival (1988) Dimension invariance of subdivision, Technical Report TR-88-30, University of Ottawa.

8. D. Kelly (1977) The 3-irreducible partially ordered sets, Canad. J. Math. 29, $367-383$.

9. H. M. MacNeille (1937) Partially ordered sets, Trans. Amer. Math. Soc. 42, 416-460.

10. S. K. Rao, P. Sadayappan, F. K. Hwang, and P. W. Shor (1990) The rectilinear Steiner Arborescence Problem.

11. E. R. Tufte (1983) The Visual Display of Quantitative Information, Graphics Press, pp. 197. 